Noboru Ito*

Nagoya Math. J.

Vol. 39 (1970), 23-28

\title{
NOTE ON THE GHARAGTERS OF SOLVABLE GROUPS
}

\author{
NOBORU ITO*
}

$\S 1$.

Let $\&$ be a solvable group of order $g$. Let $p$ be a prime and let $g=p^{a} g^{\prime}$ with $\left(p, g^{\prime}\right)=1$. In [4] we have tried to find sufficient conditions for $\mathscr{S}$ to possess an irreducible character of $p$-defect 0 , that is, a character whose degree is divisible by $p^{a}$.

The following theorem (for arbitrary finite groups) is well-known ([1], $(9 F))$.

I. If $\& 5$ possesses an irreducible character of $p$-defect 0 , then $\&$ contains no non-trivial normal $p$-subgroup.

Now what actually was proved in the proof of the main theorem in [4] (Theorem 1) is the following theorem (cf. [5]).

II. Let \&s contain no non-trivial normal $p$-subgroup. (1) If $p$ is odd and is not a Mersenne prime, then there exist two Sylow $p$-subgroups $\mathfrak{P}_{1}$ and $\mathfrak{P}_{2}$ such that $\mathfrak{P}_{1} \cap \mathfrak{P}_{2}=\mathbb{E}$. (2) (1) also holds for a Mersenne prime $p$, provided that the order of $\mathbb{E S}$ is odd. (3) (1) also holds for $p=2$, provided that every odd prime divisor $q$ of the order of \&s is not a Fermat prime and is congruent to $1 \bmod 4$. (4) (1) also holds for any prime $p$, provided that elements of order $p$ of a Sylow $p$-subgroup together with the identity forms a subgroup.

Since then Green ([2]) has proved the following theorem (for arbitrary finite groups).

III. If $\mathbb{E S}$ possesses an irreducible character of $p$-defect 0 , then there exist two Sylow $p$-subgroups $\mathfrak{P}_{1}$ and $\mathfrak{F}_{2}$ such that $\mathfrak{F}_{1} \cap \mathfrak{P}_{2}=£$. Namely, the conclusion of II, (1) holds without any restriction on $p$.

The following theorem (for arbitrary finite groups) is also well-known ([1], $(6 G))$.

Received Nov. 15, 1968.

* This work was partially supported by NSF Grant GP-6539. 
IV. If \&s possesses an irreducible character of $p$-defect 0 , then $\mathbb{S}$ contains an element $G$ of $p$-defect 0 , that is, an element $G$ the order of whose centralizer is prime to $p$.

On the other hand, the following fact is noticed in [4] (Lemma 1).

V. Let $\mathfrak{N}$ be a normal subgroup of $\mathbb{S}$, whose order is prime to $p$. If $\mathfrak{N}$ contains an element of $p$-defect 0 (in $\mathbb{S})$, then $\mathbb{S}$ possesses an irreducible character of $p$-defect 0 .

Now the primary concern of [4] was the following proposition.

(\#) Under certain circumstances the non-existence of non-trivial normal $p$-subgroups implies the existence of an irreducible character of $p$-defect 0 .

Unfortunately, in the formulation of Theorem 1 in [4] a strong condition, which is the main driving power of the induction argument in the proof of Theorem 1 in [4] is carelessly not stated.** That is the following condition.

$(\mathfrak{P} \longrightarrow p)$. Let $\mathfrak{B}$ be a fixed Sylow $p$-subgroup of $\mathbb{S}$. Then every element of $\$$, which is commutative with no element $(\neq E)$ of $\mathfrak{P}$, has $p$ defect 0 .

Now the purpose of this note is (1) to show that under the condition $(\mathfrak{P} \longrightarrow p)$, together with the condition in II securing the conclusion of II, (1), (\$) always is true, (ii) to state some conditions on the group structure under which $(\mathbb{H})$ is true without assuming the condition $(\mathfrak{P} \longrightarrow p$ ), (iii) to discuss some examples which show the necessity of the condition $(\mathfrak{P} \longrightarrow p)$, and (iv) to discuss the proof of a theorem to which Theorem 1 of [4] has been applied ([6]).

§ 2.

Proposition 1. Assume that $\mathbb{S}$ satisfies the condition $(\mathfrak{P} \longrightarrow p)$. Let $G$ be

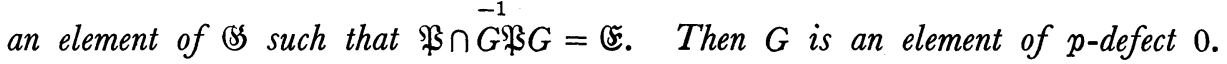

Proof. Assume that $G$ is not an element of $p$-defect 0 . Then there exists an element $H(\neq E)$ of $\& 5$ which is commutative with $G$ and has order a power of $p$. By the condition $(\mathfrak{B} \longrightarrow p)$ we may assume that $H$ belongs to $\mathfrak{P}$. Then $\mathfrak{P} \cap G^{-1} \mathfrak{B} G$ contains $H$. This is a contradiction.

Proposition 2. Assume that (S) satisfies the condition $(\mathfrak{P} \longrightarrow p)$, and that $\mathbb{S}$ contains no non-trivial normal p-subgroups, and that \&S satisfies the condition in II

** The author is indebted to Paul Fong for a valuable comment. 
.securing the conclusion of $I I,(1)$. Let $\mathfrak{F}$ be the Fitting subgroup of $\mathbb{B}$. Then $\mathfrak{F}$ contains an element of $p$-defect 0 (in $\mathbb{S}$ ).

Proof. Consider the subgroup FrF. By a theorem of Fitting ([3]) the centralizer of $\mathfrak{F}$ in $\mathfrak{S}$ is contained in $\mathfrak{F}$. Hence $\mathfrak{F P}$ contains no non-trivial normal $p$-subgroup (of $\mathfrak{F P}$ ). Therefore, by assumption, there exists an element $G$ of $\mathfrak{F}$ such that $\mathfrak{B} \cap \bar{G}^{-1} \mathfrak{P} G=\mathbb{E}$. By Proposition $1 G$ is then an element of $p$-defect 0 .

Theorem 1. Assume that \&S satisfies the condition $(\mathfrak{F} \longrightarrow p)$, that $\&$ contains no non-trivial normal p-subgroups, and that \&s satisfies the condition in II securing the conclusion of $I I,(1)$. Then \&s possesses an irreducible character of $p$-defect 0 .

Proof. By Proposition 2 it suffices to apply $V$ to $\mathfrak{F}$ and $\mathbb{S}$.

Here a sufficient condition for $\mathbb{S}$ to secure the property $(\mathfrak{P} \longrightarrow p)$ will be noticed.

Proposition 3. If a Sylow $p$-complement $\mathfrak{S}$ of $\& S$ is abelian, then \&S statisfies the condition $(\mathfrak{P} \longrightarrow p)$.

Proof. Let $G$ be an element of \&s such that $G$ has order prime to $p$ and commutes with no element $(\neq E)$ of $\mathfrak{B}$. Using P. Hall's theorem we may assume that $G$ belongs to $\mathfrak{H}$. Let $H \neq E$ be an element of $\&$ such that $H$ has order a power of $p$ and commutes with $G$. Then we may write $H=K L$, where $K$ and $L$ are elements of $\mathfrak{H}$ and $\mathfrak{P}$ respectively. Since $G H=H G$ and $G K=K G$, we obtain $G L=L G$. By assumption this implies that $L=E$ and $H=E$. This is a contradiction.

\section{§ 3.}

Theorem 2. Assume that \&s contains no non-trivial normal p-subgroup, and that $\mathbb{S}$ satisfies the condition in II securing the conclusion of $I I,(1)$. If \&s has nilpotent length 2, then 8 posesses an irreducible character of $p$-defect 0 .

Proof. By assumption there exists a nilpotent subgroup $\mathfrak{R}$ of $\mathbb{E S}$ such that $\mathbb{S} / \mathfrak{R}$ is also nilpotent. By assumption the order of $\mathfrak{N}$ is prime to $p$. Now we apply an induction argument with respect to the order of $\mathbb{S}$. If $\mathfrak{S} / \mathfrak{A}$ is not a $p$-group, $\mathscr{S}$ contains a proper normal subgroup $\mathfrak{T}$ whose index in $\mathscr{S}$ is prime to $p$. By the induction hypothesis $\mathfrak{S}$ possesses an irreducible character $\zeta$ of $p$-defect 0 . Let $\chi$ be an irreducible component of the 
character of \&s induced by $\zeta$. Then using Clifford's theorem ([3], p. 565), we see that $\chi$ has $p$-defect 0 . Thus we can assume that $\mathbb{S} / \mathfrak{N}$ is a $p$-group, that is, $\mathfrak{N}$ is a Sylow $p$-complement of $\mathbb{S}$.

Let $\Phi(\mathfrak{R})$ be the Frattini subgroup of $\mathfrak{R}$. If $\Phi(\mathfrak{R}) \neq \mathbb{E}$, then consider $(\mathscr{S} / \Phi(\mathfrak{R})$. If $\mathbb{S} / \Phi(\mathfrak{R})$ contains a non-trivial normal $p$-subgroup $\mathfrak{Q} \Phi(\mathfrak{R}) / \Phi(\mathfrak{N})$, where $\mathfrak{Q} \neq \mathbb{E}$ is a $p$-subgroup of $\mathfrak{S}$, then using Sylow's theorem get $\mathscr{S}=N(\mathfrak{Q}) \Phi(\mathfrak{N})$, where $N(\mathfrak{Q})$ is the normalizer of $\mathfrak{Q}$ in $\mathscr{S}$. This is a contradiction. Thus we may assume that $\Phi(\mathfrak{N})=\mathfrak{E}$, which implies that $\mathfrak{N}$ is abelian.

By Proposition 3 and Theorem 1 we now get Theorem 2.

THEorem 3. Assume that \&s contains no non-trivial normal p-subgroup. If (S) is metabelian, then $\mathbb{B S}$ possesses an irreducible character of $p$-defect 0 .

Proof. Take the commutator subgroup of $\mathbb{S}$ as $\mathfrak{N}$ in the proof of Theorem 2. Then we can reduce \&s to the case where the Sylow $p$-complements and Sylow $p$-subgroups are abelian.

Theorem 4. Let $\mathbb{E S}$ contain no non-trivial normal p-subgroup. If $\mathfrak{P}$ is cyclic, then \&s possesses an irreducible character of $p$-defect 0 .

Proof. Let $\AA$ be a minimal normal subgroup of $\leftrightarrow$. By assumption $\AA$ has order prime to $\mathrm{p}$. If $\leftrightarrow / \Re$ contains no non-trivial normal $p$-subgroup, then applying an induction argument to $\mathbb{S} / \Re$, we see that $\mathbb{S} / \Re$, and hence (S), possesses an irreducible character of $p$-defect 0 . So let $\mathfrak{R} / \mathfrak{R}$ be a normal subgroup of $\& / \Re$ of order $p$, where $\mathscr{Q}$ is a subgroup of $\&$ order $p$. By Sylow's theorem $\mathscr{Q}$ contains all elements of $\&$ of order $p$. Let $G$ be an element $(\neq E)$ of $\Re$. If $G$ is not of $p$-defect 0 , then the centralizer of $G$ contains some non-trivial, and hence all elements of $\mathfrak{Q}$. Thus $G$ belongs to the center of $\mathfrak{Q} \Re$. Since $\mathscr{\Omega}$ is minimal, $\mathfrak{Q}=\mathfrak{Q} \times \Re$. Therefore $\mathfrak{Q}$ is normal in $\&$. This is a contradiction. Thus $G$ has $p$-defect 0 . Now by $V$ we get Theorem 4 .

$\S 4$.

It is not difficult to construct groups which do not satisfy the condition $(\mathfrak{B} \longrightarrow p)$. The following examples show, however, that Theorems 2,3 and are also best possible.

Let $p, q$ and $r$ be distinct prime numbers such that $\frac{q^{p}-1}{q-1}=p r$. 
Examples of such triplets are $\{p, q, r\}=\{2,5,3\},\{3,13,61\},\{5,11,2331\}, \ldots$ First we notice some properties of such triplets.

(i) $q^{p} \equiv 1(\bmod r)$ and $q \neq 1(\bmod r)$.

(ii) $q \equiv 1(\bmod p)$.

(iii) $r \equiv 1(\bmod p)$.

Proof. (j) If $q \equiv 1(\bmod r)$, then $\frac{q^{p}-1}{q-1}=q^{p-1}+\cdots+q+1 \equiv p \equiv 0$ $(\bmod r)$. This is a contradiction. (ii) By Fermat's theorem $q^{p-1} \equiv 1(\bmod$ $p)$. Since $q^{p} \equiv 1(\bmod p)$, we get that $q \equiv 1(\bmod p)$. (iii) By Fermat's theorem $q^{r-1} \equiv 1(\bmod r)$. Thus by $(\mathrm{i})$ we obtain $r \equiv 1(\bmod p)$.

Let $G F(q)$ and $G F\left(q^{p}\right)$ (containing $G F(q)$ ) denote the fields of $q$ and $q^{p}$ elements respectively. Let $\sigma$ be an element of order $p$ in the Galois group of $G F\left(q^{p}\right)$ over $G F(q)$. By (i) $G F\left(q^{p}\right)$ contains a primitive $r$-th root of unity $\varepsilon$. Then

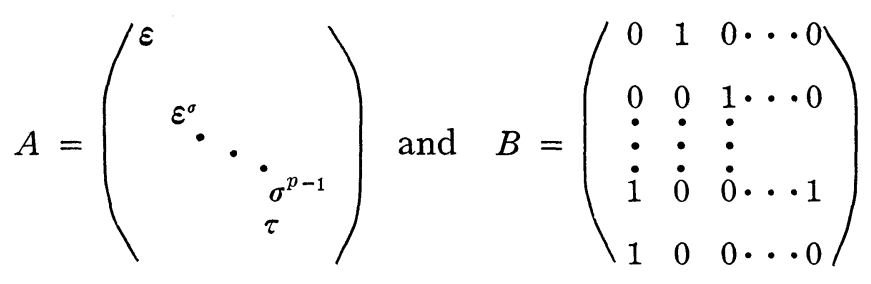

generate a non-cyclic group of order $r p$. Since the trace of every matrix of $\langle A, B\rangle$ lies in $G F(q)$, there exists a non-singular matrix $V$ with entries in $G F\left(q^{p}\right)$ such that $A^{*}=\stackrel{-1}{V} A V$ and $B^{*}=\stackrel{-1}{V} B V^{-1}$ have entries in $G F(q)$ ([3], p. 545). By (ii) $G F(q)$ contains a primitive $p$-th root of unity $\tau$. Then $A^{*}, B^{*}$ and $C=\left(\begin{array}{ll}\tau & \ddots \\ \tau\end{array}\right)$ generate a group of order $p^{2} r$, which is the direct product of $\left\langle A^{*}, B^{*}\right\rangle$ and $\langle C\rangle$. Let $\mathbb{S}$ be the split extension of the $p$-dimensional vector space $\mathfrak{B}$ over $G F(q)$ by $\left\langle A^{*}, B^{*}, C\right\rangle$.

$\mathbb{B S}$ is an $A$-group of order $p^{2} q^{p} r$. \&s has the nilpotent length 3 , and the second commutator subgroup of $\& S$ equals $\mathfrak{B}$ which is abelian. is contains no non-trivial normal $p$-subgroup.

Now we show that $\$ s$ does not possess an irreducible character of $p$ defect 0 . By IV it is enough to show that \&s does not contain an element of $p$-defect 0 .

Since the Sylow $p$-subgroups of \&s are not cyclic, there exists an element $V \neq E$ of $\mathfrak{B}$ which is commutative with an element of order $p$ ([3], 
p. 502). Since $C$ belongs to the normalizer of $\langle V\rangle$ and does not commute with $V$, the normalizer of $\langle V\rangle$ has order $p^{2} q^{p}$. If there exists an element $V_{0}$ of $\mathfrak{B}$ of $p$-defect 0 , then the normalizer of $\left\langle V_{0}\right\rangle$ has order $p q^{p}$. Since the number of subgroups of order $q$ of $\mathfrak{B}$ equals $\frac{q^{p}-1}{q-1}=p r$, every subgroup of order $q$ of $\mathfrak{B}$ must be conjugate to $\left\langle V_{0}\right\rangle$. But certainly $\langle V\rangle$ is not conjugate to $\left\langle V_{0}\right\rangle$. This shows that there exists no element of $\mathfrak{B}$ of $p$ defect 0 .

$\S 5$.

Theorem 1 of [4] has been applied to prove the following fact ([6], Proposition 2). If $\& 5$ is an $A$-group and if $G$ is an element of \&s not belonging to the Fitting subgroup $\mathfrak{F}$ of $\mathscr{S}$, then there exists an irreducible character $\chi$ of \&s such that $\chi(G)=0$. We can prove this as follows.

We use an induction argument with respect to the order of the group. Let $\mathfrak{M}$ be a minimal normal subgroup of $\mathbb{S}$ and let $\boldsymbol{F}(\mathfrak{M}) / \mathfrak{M}$ be the Fitting subgroup of $\mathbb{S} / \mathfrak{M}$. If $G$ does not belong to $\boldsymbol{F}(\mathfrak{M})$, then we can apply the induction hypothesis to $G \mathfrak{M}$ and $\mathscr{S} / \mathfrak{M}$. Hence we may assume that $G$ belongs to $\boldsymbol{F}(\mathfrak{M})$, which implies that $\boldsymbol{F}(\mathfrak{M}) \neq \mathfrak{F}$. Thus $\boldsymbol{F}(\mathfrak{M})$ has nilpotent length 2.

Now let $p$ be a prime divisor of the order of $G \mathfrak{F}$, and let $\mathfrak{P}$ be a Sylow $p$-subgroup of $\mathfrak{F}$. Then $\frac{\boldsymbol{F}(\mathfrak{M})}{\mathfrak{P}}$ contains no non-trivial $p$-normal subgroup. By Theorem $2 \frac{\boldsymbol{F}(\mathfrak{M})}{\mathfrak{B}}$ possesses an irreducible character $\zeta$ of $p$ defect 0 . Let $\chi$ be an irreducible component of the character of $\frac{\mathbb{S}}{\mathfrak{S}}$ induced by $\zeta$. Then by a theorem of Clifford ([3], p. 565) we see that $\chi \mid \boldsymbol{F}(\mathfrak{M}) / \mathfrak{S}$ decomposes into irreducible characters of $\boldsymbol{F}(\mathfrak{M}) / \mathfrak{S}$ of $p$-defect 0 . Then we get $\chi(G)=0$ ([1], 6E).

\section{BIBLIOGRAPHY}

[ 1] R. Brauer, Zur Darstellungstheorie der Gruppen endlichen Ordnung, I. Math. Zeitschr. 63 (1956), 406-444.

[2] è.A. Green, Blocks of modular representation, Math. Zeitschr. 79 (1962), 100-115.

[ 3 ] B. Huppert, Endliche Gruppen, I. Springer, Berlin-Heidelberg-New York, 1967.

[ 4 ] N. Ito, On the characters of soluble groups, Nagoya Math. J. 3 (1951), 31-48.

[ 5 ] N. Ito, Über den kleinsten p-Durchschnitt auflosbarer Gruppen, Arch. Math. 9 (1958), 27-32.

[6] N. Ito, Note on A-groups, Nagoya Math J. 4 (1952), 79-81.

Department of Mathematics, University of Illinois 